\title{
Diacronie
}

Studi di Storia Contemporanea

$N^{\circ} 25,1 \mid 2016$

"Se creare è definire"

\section{Immigrazione e contesa politico-religiosa nella Prima Repubblica: la Cappella di Sant'Antonio dei poveri a Ribeirão Preto}

\section{Wlaumir Donisete de Souza}

Traduttore: Jacopo Bassi

\section{(2) OpenEdition Journals}

Edizione digitale

URL: http://journals.openedition.org/diacronie/3965

DOI: 10.4000/diacronie.3965

ISSN: 2038-0925

\section{Editore}

Association culturelle Diacronie

Notizia bibliografica digitale

Wlaumir Donisete de Souza, « Immigrazione e contesa politico-religiosa nella Prima Repubblica: la

Cappella di Sant'Antonio dei poveri a Ribeirão Preto », Diacronie [Online], Nㅜ 25, 1 | 2016, documento 7 , Messo online il 29 mars 2016, consultato il 30 avril 2019. URL : http://journals.openedition.org/ diacronie/3965; DOI : 10.4000/diacronie.3965 


\title{
Diacronie
}

\section{Immigrazione e contesa politico-religiosa} nella Prima Repubblica: la Cappella di Sant'Antonio dei poveri a Ribeirão Preto

\author{
Wlaumir Donisete De SOUZA* \\ traduzione a cura di Jacopo BASSI
}

L'intento di questo articolo è discutere il ruolo giocato dalla figura Sant'Antonio nei conflitti tra il cattolicesimo luso-brasiliano e quello ultramontano a Ribeirão Preto, nello Stato di San Paolo. Oltre alla comprensione delle dinamiche del cattolicesimo luso-brasiliano e dell'ultramontanismo, emerge il ruolo dei nazionalismi lusitano e italiano nel contesto del processo di riforma della Chiesa cattolica nel Brasile della Prima Repubblica.

\section{La politica d'immigrazione e la pastorale dell'immigrante}

$\mathrm{I}$

l possesso ufficiale della terra brasilis fu sancito da un atto religioso. La Chiesa era del modello statale che ufficializzava il cristianesimo attraverso il padroado ${ }^{1}$. Atti ufficiali come il registro delle terre, dei matrimoni e dei decessi erano prerogative ecclesiastiche che si traducevano nella donazione di terre ai

\footnotetext{
${ }^{1}$ Il padroado consisteva in un insieme di privilegi concessi dal Papa al re del Portogallo nel XV secolo. La Santa Sede affidò ai re portoghesi l'amministrazione della Chiesa locale: ad essi competeva decidere la costruzione di chiese e la nomina di sacerdoti e vescovi. Il regno di Portogallo acquisì così sia le potestà temporali, sia quelle religiose. Questi poteri divennero presto appannaggio di uomini politici. Sul tema si veda: GONÇALVES, Nuno da Silva,"Padroado", in MOREIRA AZEVEDO, Carlos (dir. de), Dicionário de História Religiosa de Portugal, vol. III, Lisboa, Círculo de Leitores, 2001, pp. 364-368; AZZI, Riolando, História do Pensamento Católico no Brasil, Vol. I, A cristandade colonial: um projeto autoritário, São Paulo, Paulinas, 1987; DORNAS FILHO, João, O Padroado e a Igreja brasileira, São Paulo, Companhia Ed. Nacional, 1938. [N.d.T.].
} 
fini della costituzione del patrimonio ecclesiastico, nei battesimi, nella celebrazione delle nozze e nelle esequie. Il cittadino portoghese diveniva "cittadino" attraverso il battesimo.

La Chiesa in larga misura registrava le terre originariamente sottoposte alla sesmaria $^{2}$ o il cui possesso era stato legittimato con modalità diverse: ad esempio la donazione di una zolla per la creazione di una cappella che avrebbe dato origine a un villaggio e, successivamente, a una città. Benché l'accesso al possesso della terra, nella colonia come in gran parte dell'Impero, fosse facile, la definizione della proprietà diveniva quasi impossibile allorquando si desiderava portare a termine questo compito a causa del marasma di leggi e decreti esistenti; a ciò si sommavano i confini, sempre incerti e determinati dal differente livello di potere delle persone coinvolte nell'istanza.

Nell'intento di controllare la società su due fonti, la religione e la legge, il governo portoghese offriva uno spazio privilegiato dal punto di vista amministrativo - con un fondamento ideologico - alla Chiesa. Parallelamente la costruzione progressiva dello Stato equivaleva a una riduzione di spazio per la Chiesa nell'ingegneria istituzionale del governo del paese. Questa fu sancita, almeno in parte, dall'esistenza del placet nei confronti dei documenti ecclesiastici che sarebbero potuti arrivare al clero brasiliano.

Lo Stato non cercava solamente di ottenere il monopolio della forza, ma anche quello dell'emanazione delle leggi. Per questa ragione il processo di secolarizzazione seguì il suo corso revocando alla Chiesa l'attribuzione di religione di Stato, ossia di un'articolazione di quest'ultimo. La Lei de terras del 1850 rappresentò un duplice segnale dell'avanzamento di questo processo. In primis, perché stabilì il monopolio dello Stato nella legalizzazione e nella registrazione delle terre, annullando la consolidata strategia dei possessori di terre del patrimonio ecclesiastico. La donazione di terre alla Chiesa non era solamente una questione di religiosità, ma parte di un processo di legalizzazione delle stesse realizzato accaparrandosi le terre dello Stato con l'appoggio istituzionale della Chiesa, in un processo senza soluzione di continuità che aveva trasformato quest'ultima in una delle maggiori proprietarie terriere del paese.

Il controllo della proprietà e l'accesso alla terra divennero essenziali in Brasile negli anni Quaranta dell'Ottocento, di riflesso all'ampliamento del flusso migratorio europeo

\footnotetext{
${ }^{2} \mathrm{O}$ "sesta parte", era un istituto giuridico atto a normare l'assegnazione delle terre nato in Portogallo nel XIV secolo; la Lei de Terras del 1850 fu la prima a regolare la proprietà privata e a segnare la fine del sistema del regime delle Sesmarias. Sul tema: CAETANO, Marcelo, "As sesmarias no direito luso-brasileiro", in Revista do Instituto Histórico e Geográfico Brasileiro, 348, 3/1985, pp. 19-34; DINIZ, Mônica, «Sesmarias e posse de terras: política fundiária para assegurar a colonização brasileira», in Histórica, 1, 2/2005; BECK VARELA, Laura, Das Sesmarias à Propriedade Moderna: Um Estudo de História do Direito Brasileiro, Rio de Janeiro, Renovar, 2005 [N.d.T.].
} 
che doveva sostituire la manodopera schiavile. In questo quadro la Lei de terras del 1850 rappresentò un segnale del processo di rafforzamento dello Stato e del capitalismo3.

Era necessario che una parte della popolazione che si insediava non fosse in grado di imporre le proprie condizioni di lavoro e il livello di remunerazione all'imprenditore agricolo "capitalista" brasiliano. Per questa ragione si fece ricorso alla manodopera degli immigranti transoceanici - che nel proprio paese d'origine erano privi dei mezzi di produzione - e lo si fece facendone affluire un numero più che sufficiente a costituire un contingente di manodopera di riserva. Alle classi dominanti europee il processo di incentivazione legale o culturale dell'emigrazione risultava gradito per garantire la "pace sociale", mentre in Brasile lo era come strumento per fornire braccia a buon mercato per il lavoro, ma inquesto modo portava - soprattutto i neri, ma anche i bianchi discendenti di portoghesi - all'esclusione dal mercato del lavoro.

Per conseguire l'obiettivo di costituire un contingente di manodopera a buon mercato, gli agrari - che nel corso della storia del Brasile furono e rimasero fortemente legati agli orientamenti politici del paese -, intraprese l'elaborazione di un progetto di sostegno all'immigrazione; questo sarebbe stato reso possibile grazie all'unione delle forze private capitaliste e avrebbero cercato nello Stato l'appoggio necessario per velocizzare questo processo. Venne elaborato un progetto di sovvenzionamento dell'immigrazione che diede la priorità agli Italiani per via del fatto che gli venivano attribuite, fra le altre caratteristiche, quelle di essere cattolici, morigerati e amanti della famiglia, questo almeno secondo la percezione dell'epoca. Questo avvenne anche nel caso della regione dell'attuale Stato di San Paolo, orientamento che fu successivamente seguito anche da altri Stati ${ }^{4}$.

Grazie al sovvenzionamento il numero di immigrati che entrarono nel paese aumentò in una misura mai vista prima in Brasile: neppure il periodo della ricerca dell'oro a Minas Gerais, vide un simile incremento del fenomeno. Essendosi costituito questo contingente, il fazendeiro finì per avere a disposizione le condizioni necessarie per fare pressione - in realtà commettere abuso - sui lavoratori utilizzando questa situazione come elemento di conservazione della mentalità e del potere schiavocratico ${ }^{5}$.

\footnotetext{
3 SILVA, Ligia Maria, Terras devolutas e latifúndio, Campinas, UNICAMP, 2008.

4 De SOUZA, Wlaumir Doniseti, Anarquismo, Estado e pastoral do imigrante, São Paulo, UNIESP, 2000.

5 GARCIA, Maria A. M., Trabalho e resistência: os trabalhadores rurais na região de Ribeirão Preto (1890-1920), Tesi di Specializzazione - Faculdade de História, Direito e Serviço Social dell'Universidade Estadual Paulista, Franca, 1993.
} 
Quale condizione necessaria per lo sfruttamento del lavoratore, volutamente confuso con lo schiavo nelle fazende, i politici si abbandonarono ad una retorica che vedeva nella mancanza di braccia per il lavoro la necessità di un'estensione, apparentemente indefinita nel tempo, della sovvenzione all’immigrazione. A ciò si doveva assommare la concorrenza degli stranieri con i lavoratori nazionali, soprattutto i discendenti dei portoghesi, che vedevano ridursi le possibilità di trovare impiego.

La disputa tra lavoratori autoctoni e quelli stranieri per il lavoro toccava l'orgoglio dei primi. Presentati come morigerati, amanti della famiglia, disciplinati, cattolici - tra le tanti attribuzioni -, gli immigrati venivano preferiti per il lavoro mentre gli autoctoni erano messi da parte perché considerati lenti, indolenti e indisciplinati.

L’immigrato appariva come il proletario per eccellenza: era privo dei mezzi di produzione, dei legami di amicizia e parentela allargata che garantivano maggior potere, senza grandi opportunità - almeno inizialmente - di organizzarsi politicamente e ideologicamente per difendere i propri interessi economici dal momento che era soggetto in ogni momento ad una possibile espulsione. Questo quadro trovava la sua antitesi nell'anarchismo e nel sindacalismo.

Parallelamente alla tendenza a considerare l'immigrante come lavoratore ideale, le élites, preoccupate di identificarsi con la civilizzazione europea, si curavano di educare i propri figli in collegi cattolici amministrati e composti da professori e professoresse di un'altra nazionalità, principalmente francese, consolidando così l'antico progetto di Stato imperiale in cui il controllo ideologico sarebbe stato esercitato dalla religione ${ }^{6}$.

Per l'élite nazionale tutto doveva essere straniero: dal lavoratore delle piantagioni di caffé - passando per la moda e l'educazione - fino alla decorazione e all'architettura delle abitazioni. L'orgoglio nazionale era inesistente tra queste classi e, per di più, si attribuiva il carattere di cui si è accennato più sopra al lavoratore "autoctono", che si vedeva - al pari di Carlota Joaquina7, ai primordi dell'Impero - non solamente da un punto di vista legale, ma anche di fatto, in esilio dal mondo civilizzato.

In questo quadro di costante e crescente valorizzazione della società europea, che nel confronto con il Brasile faceva sorgere un etnocentrismo peculiare delle classi dirigenti e dominanti, l'immigrante italiano - europeo e cattolico - dominava la società

\footnotetext{
6 MANOEL, Ivan, Igreja e educação feminina, Tesi di dottorato - Programa de Pós-Graduação em História - Faculdade de Filosofia Letras e Ciências Humanas da Universidade de São Paulo, São Paulo, 1988.

7 Carlota Joaquina di Borbone, moglie di João VI di Braganza, fu imperatrice del Brasile fra il 1825 e il 1826 [N.d.T.].
} 
del lavoro perché veniva considerato superiore a quello autoctono, meticcio e indolente, che era gravato - secondo i preconcetti dell'epoca - dal clima tropicale di quella terra ${ }^{8}$.

In quanto elemento aggregante di questi aspetti culturali, la Chiesa cattolica, nell'ambito dell'avvio di una relazione dialettica con lo Stato moderno, dava avvio alla rivendicazione a livello mondiale - consolidata dal Concilio Vaticano I del 1870 quale prosecuzione del Concilio di Trento - dell'autonomia della Chiesa nei confronti dello Stato, accentuando ciò che era definito ultramontanismo ${ }^{9}$.

L'ultramontanismo, come è stato spiegato molto bene da Augustin Wernet ${ }^{10}$, aveva come obiettivo la centralizzazione delle decisioni politico dottrinarie a partire dall'infallibilità papale. Una delle bandiere dell'ultramontanismo era la moralizzazione e la catechizzazione della società come se si trattasse di un tuttuno: nella società era incluso lo Stato, cioè la servitù dello Stato secondo l'istituto del Padroado, la Signora delle Nazioni nell'ultramontanismo.

Uno dei cardini dell'ultramontanismo era l'educazione - intesa come indottrinamento religioso - dall'infanzia sino alla vita adulta. Sui banchi delle scuole cattoliche, responsabili dell'educazione dell'élite nazionale dal momento che la rete educativa pubblica si era ridotta anche per via dell'assenza di personale qualificato, si cercava a garantirsi la possibilità di esercitare influenza sui politici del futuro. La Chiesa, per parte sua, si preoccupava di formare una parte del suo clero e i frati finivavo per risultare gli educatori privilegiati di quell'epoca.

Potendo educare i figli dell'élite politica ed economica del paese, la Chiesa, riuscì a influire sulle coscienze in grado di decidere le politiche del Brasile. Ebbe così luogo nella Camera dei Deputati un dibattito sulla scelta della tipologia di immigranti di cui privilegiare l'ingresso nel paese. L'orientamento dell'immigrazione oltre a contribuire all'embranquecimento della nazione - l'“imbiancamento", privilegiando coloni bianchi - avrebbe consolidato il modello culturale ereditato dal sistema coloniale: essere cattolico era un elemento basilare, oltre che fondante, della nazionalità.

Il flusso immigratorio italiano rispondeva ai principali orientamenti espressi dalle classi dirigenti: incrementare il numero di bianchi nella popolazione brasiliana e conservare l'unità religiosa del paese rendendo possibile la costituzione di un ampio contingente di braccia disponibili per il lavoro, tutto ciò assieme al conseguimento

8 RIBEIRO, Maria Therezinha Janine, Desejado e temido: preconceito contra o imigrante italiano na Primeira República, Programa, Testi di specializzazione - Corso di Laurea in Storia - Faculdade de Filosofia Letras e Ciências Humanas da Universidade de São Paulo, São Paulo, 1985, p. 104.

9 De SOUZA, Wlaumir Doniseti, op. cit.

${ }^{10}$ WERNET, Augustin, A Igreja paulista no século XIX, São Paulo, Ática, 1987. 
dell'“europeizzazione” della nazione. Questa era la figura dell'emigrante sovvenzionato dallo Stato di San Paolo.

Di fronte all'immigrazione, la Chiesa, determinata a portare avanti una percezione di sé e la visione del mondo ultramontana, nel 1887 creò una congregazione, gli Scalabriniani o Carlisti, che si occupava specificamente della pastorale degli immigrati italiani in America. Definiti missionari di San Carlo Borromeo dal loro fondatore, don Giovanni Battista Scalabrini, sfruttarono il particolare interesse che la curia romana dimostrava contribuendo alla pubblicazione della Rerum Novarum (1891), facendo in modo che questa fosse preceduta dalla lettera apostolica - anch'essa firmata da Leone XIII - che sin dal titolo evidenziava un progetto espansivo: ai nostri venerabili fratelli, gli arcivescovi e i vescovi dell'America: raccomandazioni perché proteggano gli italiani che immigrano in America (1888) ${ }^{11}$.

Nella lettera ai nostri venerabili fratelli, gli arcivescovi e $i$ vescovi dell'America: raccomandazioni perché proteggano gli italiani che immigrano in America, Leone XIII presentava i missionari di San Carlo Borromeo, conosciuti anche come scalabriniani, come i principali responsabili della pastorale degli emigranti in America, conferendo loro l'attribuzione di apostolici, il che implicava una serie di privilegi e diritti tra i quali la libera circolazione attraverso le differenti parrocchie e diocesi liberandoli dal controllo immediato dei vescovi locali.

Considerati come legittimi ultramontani e romani, gli scalabriniani finirono per ottenere, a partire dal loro arrivo in Brasile nel 1895, uno spazio senza pari. Questo dal momento che buona parte della popolazione del principale stato repubblicano almeno dal punto di vista economico - San Paolo, era costituita da immigrati italiani e di altre nazionalità europee. La pastorale dell'emigrante portava con sé una commistione tra il mantenimento e l'ampiamento della fede ultramontana degli italiani e il sentimento di appartenza alla "nazionalità" di origine.

\section{La disputa nello spazio sacro: tra il portoghese e il romano}

La cappella di Sant'Antono "dei poveri”, fondata il 7 ottobre del 1897 nella periferia di Ribeirão Preto nel quartiere di Barracão, vide il via dei lavori il 7 giugno del 1899 grazie alla sollecitudine del vice-console del Portogallo a Ribeirão Preto, Alfredo Vianna Pinto de Souza $^{12}$. La costruzione proseguì nei primi anni del XX secolo; dal 15

\footnotetext{
${ }^{11}$ Ibidem

12 ADDLS, busta Ribeirão Preto (I). 
settembre del 1902 la celebrazione dell'eucarestia fu permessa «in un chalet vicino alla cappella in costruzione» che venne benedetto il 27 novembre di quello stesso anno ${ }^{13}$.

Fondata da un uomo di origine portoghese, Alfredo Vianna Pinto de Souza con la collaborazione della famiglia Junqueira, rappresentante di spicco dell'oligarchia regionale - anch'essa di origine portoghese, ma proveniente da Minas Gerais - nella persona di Manoel Maximiano Junqueira, presidente della Camera municipale, la cappella di Sant'Antonio "dei poveri” era il luogo per eccellenza del cattolicesimo portoghese tradizionale, popolare e festoso di Ribeirão Preto.

La denominazione "dei poveri” legata a Sant'Antonio cercava di dissimulare il significato reale della figura di Sant'Antonio da Lisbona: un forte rappresentante della devozione del cattolicesimo lusitano in Brasile, assieme a San Sebastiano, e che si trovava in opposizione diretta al santo di Padova, che portava con sé la rappresentazione del cattolicesimo ultramontano-romano "all'italiana".

L'epiteto “dei poveri” enfatizzava un altro aspetto della personalità di Sant'Antonio, nato a Lisbona e morto a Padova: quello di procacciatore di pani per far cessare la fame. In una regione che concentrava la popolazione povera di origine italiana questa attribuzione "dei poveri" risultava di buon auspicio per la conquista e il mantenimento dei fedeli.

Legato alle pratiche di un cattolicesimo laico, popolare e festoso, comune al periodo coloniale e a quello imperiale, Sant'Antonio da Lisbona era portatore - per i portoghesi - di una vigorosa rappresentazione della lotta contro l'infedele, l'indigeno, e, soprattutto, lo straniero, invasore delle terre assoggettate dai portoghesi; la benedizione conferita dalla Chiesa ai soldati, infatti, la si faceva davanti alla sua immagine. A rafforzare questa rappresentazione c'era il fatto che Antonio da Lisbona evocava una "dimensione amorosa" che univa «antichi rituali millenari dedicati al culto della fertilità, con un'evidente connotazione sessuale» e da cui aveva ricavato l'attributo di "pronubo"14.

Mentre i portoghesi e i loro discendenti veneravano il Sant'Antonio nato a Lisbona, l'immigrante italiano, al contrario, in quanto straniero venerava quel Sant'Antonio che morì a Padova. Benché si trattasse dello stesso uomo, il santo, attraverso le sue differenti denominazioni, esprimeva le sue multiple personalità. Lo scontro tra $\mathrm{i}$ discendenti dei Portoghesi e quelli degli Italiani verteva sul mantenimento della denominazione di santo di Lisbona, connesso con il cattolicesimo del Padroado e della

\footnotetext{
${ }^{13}$ ADDLS, libro delle parrocchie (8-2-23), p. 47.

${ }^{14}$ AZZI, Riolando, A Igreja e os Migrantes, vol. II, São Paulo, Paulinas, 1988.
} 
festa, in opposizione a quello italiano legato al Santo di Padova, una devozione di stampo ultramontano che esigeva i sacramenti e la gerarchia imposta da Roma.

Sant'Antonio da Padova, in Italia, era una versione ultramontana di quello di Lisbona, mentre in Portogallo, era espressione del Padroado. Per questa ragione il santo proposto dall'ultramontanismo era portatore di una posizione mediata dal prete, che si legava alla ricezione dei sacramenti. Il suo culto si diffuse nella regione del Veneto nel XIX secolo, in un'area fortemente ultramontana e romanizzata, da dove proveniva uno dei maggiori contingenti di immigrati diretti in Brasile.

Il nocciolo della questione ruotava intorno al fatto che Sant'Antonio - per il fedele devoto - fosse di Padova o di Lisbona, quindi una questione di nazionalità legata al fatto che questi fosse portoghese o italiano, benché questa scelta riflettesse un'orientamento che si trovava o meno sotto l'influenza ultramontana. Dal momento che la Chiesa aveva privilegiato, sino a quel momento, il suo orientamento ultramontano, l'enfasi posta sulla pastorale dell'immigrante italiano da parte degli Scalabriniani, finiva talvolta per scontrarsi con i sentimenti patriottici dei Portoghesi a vantaggio degli Italiani.

Il mantenimento del Padroado, in Portogallo, rese difficile l'espansione dell'ultramontanismo in quell'area e nelle sue colonie nella misura in cui in Italia, culla della Chiesa, l'ultramontanismo si diffuse attraverso la cultura popolare e il Papa venne considerato infallibile a prescindere dall'ufficializzazione del dogma da parte del Concilio Vaticano I, nel 1870.

La devozione nei confronti di Sant'Antonio era permeata dai sentimenti di identità nazionale e questo collocava le parti in contrapposizione di fronte agli usi, ai costumi e alle posizioni che sarebbero state adottate e seguite nella società, dal momento che essere legati al Santo di Padova significava sposare una posizione ultramontana. Questo poteva condurre a una pratica sociale di avversione ufficiale nei confronti della politica, dello Stato e del mondo moderno, a un'accettazione del prete come unico leader "spirituale" delle coscienze e monopolizzatore del sacro per via del fatto che celebrava $\mathrm{i}$ riti, escludendo di fatto i colonnelli e gli altri proprietari locali dal controllo della Chiesa.

Al Santo di Lisbona corrispondeva, invece, una pratica sociale del cattolicesimo dove il laico era colui che orientava il tono del rito: la celebrazione svolgeva un ruolo fondamentale e politicamente importante, legittimando il prete solamente a scegliere quella che i cattolici della parrocchia consideravano come la "forma migliore". In altre parole, il culto di Sant'Antonio di Lisbona era una delle forme di resistenza delle 
antiche confraternite e fratellanze, in cui il prete era visto come nulla più che un impiegato che doveva obbedienza al proprietario della cappella o della chiesa.

Considerato diversamente dai cattolici di origine portoghese o dagli immigranti italiani, Sant'Antonio si legava ai primi in quanto rappresentante di una religiosità avversata dalla Chiesa sin dall'elezione al soglio di Pio IX, nella misura in cui, invece, per il secondo gruppo portava con sé l'ideale di devozione così come la gerarchia ecclesiastica cercava di introdurlo in Brasile e, nel caso di San Paolo, come lo fece a partire dalla riforma della riforma di Don Antônio Joaquim de Mello, nella metà del XIX secolo ${ }^{15}$.

Imbevuto di cattolicesimo lusitano e del sistema del Padroado - dove le cappelle erano proprietà private o delle fratellanze e confraternite che soggiogavano i preti ai loro dettami -, secondo questo principio, Manoel Maximiano Junqueira affidò al viceconsole Alfredo Vianna de Souza il terreno per la costruzione della cappella. Tuttavia, secondo la documentazione, dopo essere stata conclusa, Vianna de Souza andò a donare l'edificio religioso al seggio vescovile.

La cappella era stata eretta nel rispetto della tradizione portoghese. Parte del gruppo dirigente strumentalizzava i sentimenti religiosi per rafforzare la sua immagine e l'influenza assieme alla popolazione di onesti benefattori, come mezzo per nascondere le atrocità commesse durante le contese elettorali e mantenersi al potere oltre a ridurre la violenza praticata sul campo.

La disputa su Sant'Antonio da Lisbona condusse la Chiesa ad affrontare l'élite locale di origine lusitana, che era proprietaria delle terre e politicamente influente, a vantaggio degli immigrati italiani che vantavano fra loro pochi membri socialmente emergenti nelle attività commerciali e liberali della città, senza che questo li collocasse in modo generalizzato, in cariche strategiche dal punto di vista politico. Tuttavua queste finivano per rappresentare un contingente di popolazione significativo,, il che non era molto in un'epoca in cui la Repubblica era oligarchica e sostituita dai discendenti dei portoghesi, il liberalismo era permeato da una cultura schiavocratica e dipendente dagli incentivi dello Stato.

In questo modo le relazioni tra il clero locale - di orientamento ultramontano e romanizzatore, che apprezzava la devozione degli Italiani e che cercava di portare avanti il progetto di divisione della diocesi di San Paolo e la scelta di Ribeirão Preto come nuova sede diocesana - e Vianna de Souza - quale rappresentante dell'élite di origine lusitana e tradizionale, detentrice del potere in ambito locale - presero un andamento sempre più teso e conflittuale.

15 WERNET, Augustin, A Igreja paulista no século XIX, São Paulo, Ática, 1987. 
Gli animi si incendiarono dal momento che la costruzione della cappella fu portata avanti da Vianna de Souza, senza la che fosse stata costituita alcuna commissione organizzatrice, e tutto il denaro riscosso a nome di Sant'Antonio, nella e per la cappella in questione, destinato al materiale di costruzione e al pagamento dei lavoratori e del pane dei poveri - che era distribuito nella cappella -, veniva speso senza alcun tipo di controllo.

Per padre Euclides Gomes Carneiro, rappresentante presso la cappella della chiesa madre di San Sebastiano, distante da questa tra uno e due chilometri circa, il denaro sarebbe dovuto essere inviato alla cappella dell'antica Santa Casa della Misericordiaa ${ }^{16}$, che sarebbe stata la sede provvisoria della parrocchia fin quando non fosse stata completata la chiesa madre, dal momento che il canonico Joaquim Antônio Siqueira, vicario di Ribeirão Preto, aveva permesso la demolizione di quella antica, il 30 agosto 1904 ${ }^{17}$. La costruzione della cappella di Sant'Antonio concorreva direttamente con quella della chiesa madre - e futura cattedrale - nella riscossione delle risorse finanziarie. La disputa fra Portoghesi e Italiani - ultramontani e romani - passava attraverso la questione economica e quella del progetto di fare di Ribeirão Preto una sede diocesana.

La preoccupazione di mantenere il controllo finanziario delle donazioni realizzate in favore di Sant'Antonio, evidenzia come quest'ultimo avesse finito per accaparrarsi un numero maggiore di devoti rispetto a San Sebastiano. D'altra parte l'assenza della costituzione di una commissione, che sarebbe servita per procacciare fondi e controllare le finanze che restavano sotto il controllo di Vianna de Souza senza essere soggette ad alcuna supervisione ecclesiastica, si palesa come resistenza del cattolicesimo lusitano. Per superare questa libertà laica nelle questioni religiose, padre Euclides sospese la celebrazione eucaristica della domenica nella cappella di Sant'Antonio “dei poveri”, centralizzandola nella Cappella della Santa Casa, annunciando la misura per il tramite del giornale locale ${ }^{18}$.

Reagendo al controllo ecclesiastico, il viceconsole Souza offrì agli Agostiniani la possibilità di celebrare nella cappella di Sant'Antonio, ma questi rifiutarono - secondo Vianna de Souza - per timore di incorrere nelle rappresaglie dell'influente padre Euclides. Gli Agostiniani sostenevano di ignorare l'origine degli attriti, tuttavia,

\footnotetext{
${ }^{16}$ ADDLS, Busta Ribeirão Preto (II). Lettera di Alfredo Vianna Pinto de Souza al vicario generale e canonico Antônio Pereira Reimão, Ribeirão Preto, del 13 settembre 1905. Lettera di Alfredo Vianna Pinto de Souza a padre Antônio Pereira Reimão, Ribeirão Preto, del 20 settembre 1905 ${ }^{17}$ ADDLS, Registro delle ordinanze e ulteriori atti ufficiali (1904-1915), p. 2.

${ }^{18}$ ADDLS, Busta Ribeirão Preto (II). Lettera di Alfredo Vianna Pinto de Souza al vicario generale e canonico Antônio Pereira Reimão, Ribeirão Preto, del 13 settembre 1905.
} 
preferivano celebrare solamente con il permesso del vescovo, dimostrando con questo come fossero legati ai principi romani e ultramontani. Così gli Agostiniani, nella persona di frate Gregorio ${ }^{19}$ rifiutavano di esaudire la richeista del viceconsole senza entrare in attrito con lo stesso e il gruppo che rappresentava, non violando l'autorità del vicario e comunicando la decisione a padre Euclides $^{20}$. Souza sollecitò tuttavia la concessione del permesso attraverso la mediazione del vicario generale, Antônio Pereira Reimão, affinché i frati potessero celebrare, confermando che una volta conclusa la costruzione avrebbe consegnato il luogo di culto al vescovo di San Paolo, Don José Camargo Barros ${ }^{21}$.

In questo gioco di potere le forze ecclesiastiche andavano convergendo intorno alla cerchia del vicario di Ribeirão Preto, il canonico Joaquim Antônio Siqueira, a scapito del viceconsole del Portogallo, Alfredo Vianna Pinto de Souza. In altre parole il rafforzamento dei principi ultramontani e della romanizzazione a detrimento del cattolicesimo popolare luso-brasiliano e del controllo della Chiesa da parte dell'oligarchia locale, finì per coincidere con l'ampliamento del potere di influenza, controllo e decisione del clero di fronte ad una delle famiglie più potenti di São Sebastião do Ribeirão Preto, gli Junqueira.

Per questa ragione padre Euclides Gomes Carneiro scrisse a Monsignor Reimão il 13 di settembre del 1905; considerava inopportuna la posizione di coordinatore della cappella di Sant'Antonio assunta al cospetto del vicario dal viceconsole Souza, dal momento che questi non rispettava l'autorità ecclesiastica dello stesso, poiché questi non aveva accettato padre João Grippa, che era stato designato per amministrare la cappella ${ }^{22}$.

La crescita economica della città di Ribeirão Preto aveva portato il potere locale a ottenere un certo grado di autonomia in ragione della distanza dalla capitale, delle difficoltà di comunicazione e per l'inutilità - dal punto di vista produttivo - di una relazione di interdipendenza con le succursali del potere centrale: lo Stato, il Partito Repubblicano Paulista e la Curia diocesana. Rendere flessibili e negoziare le decisioni con questi gruppi era la condizione necessaria per mantenere il potere in una città che cresceva sotto numerosi punti di vista, tra cui quello economico, demografico e politico.

\footnotetext{
19 ADDLS, Busta Ribeirão Preto (II). Lettera di Alfredo Vianna Pinto de Souza al canonico Antônio Pereira Reimão del 24 settembre 1905.

${ }^{20}$ ADDLS, Busta Ribeirão Preto (II). Lettera di Alfredo Vianna Pinto de Souza a padre Antônio Pereira Reimão del 20 settembre 1905.

${ }^{21}$ ADDLS, Busta Ribeirão Preto (II). Lettera di Alfredo Vianna Pinto de Souza al vicario generale Antônio Pereira Reimão, Ribeirão Preto, del 13 settembre 1905.

${ }^{22}$ ADDLS, Busta Ribeirão Preto (II). Lettera di Euclides Gomes Carneiro al canonico Antônio Pereira Reimão del 13 settembre 1905.
} 
Lo scontro tra il vice console Alfredo Vianna Pinto de Souza e il vicario di Ribeirão Preto, il canonico Joaquim Antônio Siqueira e Padre Euclides Gomes Carneiro evidenziala disputa tra la Chiesa, ultramontana e romana, e una parte della famiglia Junqueira, rappresentata in questo caso da Manoel Maximiano Junqueira, donatrice dell'area di edificazione.

La disputa era nata perché Souza si era opposto al fatto che il Cappellano della Santa Casa, padre Euclides, si appropriasse delle offerte e delle donazioni dei devoti quando egli stesso celebrava nella cappella di Sant'Antonio. A lui sarebbe dovuto spettare - secondo Vianna de Souza - solamente ciò che veniva raccolto durante la messa dal momento che la cappella era ancora in costruzione. Reagendo direttamente contro padre Euclides Gomes Carneiro affermò di pubblicare sempre il bilancio della cappella e che il prete desiderava intromettersi in tutte le questioni mentre diveniva monopolizzatore dei problemi ecclesiastici ${ }^{23}$. Le caratteristiche evidenziate come negative dal viceconsole rientravano nel processo di consolidamento della romanizzazione e dell'ultramontanismo ed erano, pertanto, positive agli occhi di Reimão e del Vescovo di San Paolo.

Gli scontri culminarono nel 1905 con l'appropriazione della cappella di Sant'Antonio da parte del sacrestano della chiesa madre ${ }^{24}$. Con questo procedimento si cercava di mostrare come il luogo di culto cattolico fosse sotto il controllo e l'amministrazione del clero e non dei laici, benché fossero benefattori della Chiesa o ricoprissero incarichi di prestigio nello Stato repubblicano laico, fosse esso brasiliano o portoghese.

Il giorno 24 di settembre del 1905, Vianna de Souza comunicò a Reimão che l'appropriazione della cappella affinché vi potesse essere celebrata la messa dal cappellano della Santa Casa della Misericordia di Ribeirão Preto, veniva da lui considerata una profanazione, una mancanza di rispetto, un'azione degna dei nemici della religione, che metteva in risalto la decadenza in cui si trovavano i religiosi e la religione.

Di fronte al fatto il viceconsole Souza affermava di aver sollecitato i giornali perché non pubblicassero nulla sulla faccenda, ma che non poteva fare alcunché riguardo alla voce del popolo, che commentava e prendeva posizione. Rinsaldò la sua posizione di fronte a Padre Euclides riaffermando di considerare se stesso come protettore della Santa Casa e benefattore di questa, il ché si trasformava in un appoggio alla costruzione

${ }^{23}$ ADDLS, Busta Ribeirão Preto (II). Lettera di Alfredo Vianna Pinto de Souza a padre Antônio Pereira Reimão del 20 settembre 1905.

${ }^{24}$ ADDLS, Busta Ribeirão Preto (II). Lettera di Alfredo Vianna Pinto de Souza al vicario generale Antônio Pereira Reimão, del 24 settembre 1905. 
della nuova chiesa madre a detrimento dell'edificio religioso eretto in onore di Sant'Antonio "dei poveri”, traducendosi in opposizione, resistenza e oppressione da parte della gerarchia ecclesiastica locale alla costruzione della cappella. Malgrado tutto, Souza garantiva che avrebbe portato avanti la diffusione del «culto di Sant'Antonio a Ribeirão Preto» ${ }^{25}$.

Dall'insistenza di Vianna nel ricorrere all'aiuto di Reimão, detentore di un incarico strategico nella curia di San Paolo, possiamo concludere che questi possedeva tatto e diplomazia sufficienti al punto da non evidenziare come la posizione del vescovo di San Paolo, Don José de Camargo Barros, fosse contraria a quella di Vianna. Reimão cercava, senza prendere posizione, di guadagnare la fiducia del viceconsole, mantenendo i rapporti con il gruppo che si sentiva legato alla Chiesa, senza arrivare a uno scontro diretto con l'istituzione. L'opposizione dei Portoghesi nei confronti del clero si sarebbe potuta concludere a danno della Chiesa, che avrebbe potuto perdere, indirettamente, l'appoggio di una delle famiglie più potenti della regione, gli Junqueira, in un'area pioniera della grande espansione prodotta dall'economia caffearia.

Nella convergenza di forze in seno al clero locale si osserva come gliAagostiniani finirono per opporsi a Souza prendendo le parti della gerarchia ecclesiastica al punto che frate Celedonio Matos scrisse al vescovo parlando di Vianna de Souza come ad un intransigente, irrispettoso dell'autorità ecclesiastica. Celedonio si opponeva all'autorità di questo laico nella costruzione della chiesa di Sant'Antonio. I guadagni procacciati nella cappella "dei poveri" toccavano anche agli Agostiniani, aldilà delle dispute tra ultramontani e lusitani. Frate Celedonio considerava tuttavia la necessità di agire con prudenza nei confronti dei provvedimenti che si sarebbero dovuti prendere dal momento che il viceconsole rappresentava un intero gruppo ${ }^{26}$, quello dei portoghesi e del cattolicesimo popolare ereditato dal padronato.

Tuttavia gli animi si inasprirono e l'appropriazione della cappella venne considerata dagli ultramontani come il compimento del dovere del prete; e questo benché ai Portoghesi l'azione apparisse come un affronto, una mancanza di rispetto e, perché no, un invito allo scontro politico tra le forze in causa: da una parte l'élite portoghese e, dall'altra, il clero filo romano, in sostegno degli Italiani. Malgrado il conflitto - e l'origine dello stesso - si riconosceva l'importanza dell'opera antoniana avviata da Vianna de Souza, che irreggimentava un grande numero di fedeli e devoti, oltre alle

\footnotetext{
25 ADDLS, Busta Ribeirão Preto (II). Lettera del viceconsole del Portogallo Alfredo Vianna de Souza a Antônio Pereira Reimão, del 20 settembre 1905.

${ }^{26}$ ADDLS, Busta Ribeirão Preto (II). Lettera di frate Caledonio Matos al vescovo Don José de Camargo Barros del 27 settembre 1905.
} 
risorse finanziarie, in uno scontro tra l'élite portoghese e gli ultramontani e filo romani, che patrocinavano la costruzione delle futura cattedrale.

La cappella - situata nella periferia di Ribeirão Preto, secondo lo stesso documento in un'area conosciuta come Barracão e densamente popolata ${ }^{27}$, era il luogo in cui si ritrovavano i coloni provenienti dall'Hospedaria dos imigrantes ${ }^{28}$ della capitale quando lasciavano la città - portò a un'accesa disputa intorno al suo controllo.

Con l'ultramontanismo il prete indicava l'orientamento ai fedeli e controllava le tematiche ecclesiastiche, così come nel cattolicesimo portoghese il colonnello o, nel caso specifico, il viceconsole - ossia i laici socialmente, politicamente ed economicamente importanti - fornivano l'orientamento che doveva essere seguito dalla Chiesa a dai suoi fedeli. Oltre a queste ragioni emergeva alla base della questione, la disputa politico-religiosa per il possesso della cappella in quanto opportunità di controllare uno dei maggiori quartieri della città, con un gran numero di fedeli - il quartiere degli immigrati proletari - indirizzandoli verso una specifica influenza cattolica e, conseguentemente, verso una determinata posizione politica.

In questo scontro di forze la tendenza ultramontana finì per sovrapporsi a quella luso-brasiliana, benché quet'ultima tendesse a riemergere. $\mathrm{Fu}$ in quest'ottica che il viceconsole del Portogallo a Ribeirão Preto, Alfredo Vianna de Souza rispose alla lettera inviata dal vicario generale Antônio Pereira Reimão, datata 28 settembre 1905, il 30 dello stesso mese.

Nella missiva affermava di avere destinato le donazioni della domenica alla Santa Casa e di aver affidato le chiavi della cappella di Sant'Antonio "dei poveri” al cappellano italiano, João Grippa, riconoscendo che «gli Italiani sono devotissimi di Sant'Antonio»29, senza far menzione del fatto che fosse di Padova. Tuttavia aveva mantenuto la custodia delle chiavi originali della chiesa, delle cassettiere (chiuse) e dei paramenti, che appartenevano al Colonnello Manoel M. Junqueira, e comunicava che se il cappellano avesse tentato di forzare le serrature dei cassetti, come aveva fatto con la cappella, questa volta avrebbe utilizzato tutta la sua influente autorità per far inteventire la polizia ad arrestarlo. Nel suo avvicinamento ai principi romanizzatori, Souza inviava una copia della lettera al vescovo e pubblicava il bilancio dell'opera antoniana, venendo spinto a questo compito da «Don Antônio Barroso, vescovo di

\footnotetext{
27 Ibidem.

${ }^{28}$ Gli hospedaria dos imigrantes erano strutture in cui venivano accolti coloro che immigravano in Brasile; da queste strutture venivano poi inviati nelle fazende e negli insediamenti coloniali [N.d.T.].

29 Di fronte allo straniero l'opposizione portoghese era tale da portare de Souza a evitare che fosse il clero italiano a controllare la chiesa, che era una proprietà privata, ma si percepisce che si andava oltre questa visione, per lo meno a livello ufficiale.
} 
Porto e ambasciatore del Vaticano». Il Portogallo si arrendeva all'ultramontanismo e, al contempo, mostrava i legami politici che avevano reso possibile la sua resistenza sino a quel momento.

La vicinanza di Vinna de Souza alle gerarchie della Chiesa trova il suo miglior riscontro nella lettera inviata dal cardinale segretario del Papa, da Roma, il 13 di febbraio del 1902, numerata come 68.215, che comunica la concessione della benedizione papale alla sua famiglia e a sua madre in virtù della propaganda in favore della devozione antoniana ${ }^{30}$, che a quell'epoca - quella della fondazione della cappella - non causava problemi alla gerarchia locale.

La lettera inviata da Reimão a Vianna de Souza avviava un processo nel quale questi si sottometteva al clero locale, avvicinandosi all'ideale romanizzatore e ultramontano, pur restando riluttante ad accettarne alcuni aspetti. In una lettera del 4 ottobre, reclamava l'adozione di misure per evitare il desigillamento della cappella e sollecitava la vigilanza nella costruzione della nuova chiesa madre, sul cantiere e sul comportamento di padre Euclides che «non celebrava messa da tre mesi», così come sulla Legião Brasileira ${ }^{1}$.

Dando ai suoi oppositori vittoriosi una dose di ciò che aveva provato, il viceconsole si convinse ad adottare i principi ultramontani per rivalersi su coloro che lo avevano attaccato. Cosciente del ruolo che doveva essere svolto dal prete e dell'aspetto centrale rappresentato dalla celebrazione eucaristica che sollecitava una vigilanza «sui topi della chiesa» che non celebravano la messa da mesi ${ }^{32}$; denunciava e attaccava direttamente padre Euclides Gomes Carneiro. Utilizzando le armi proprie della Chiesa ultramontana, sferrò il suo ultimo attacco, per effetto del quale padre Euclides avrebbe dovuto rispondere del suo comportamento davanti al vescovo, che era filoromano.

In compenso aprì la cappella al cappellano della Santa Casa affinché celebrasse di domenica e a causa della «avidità e vanità altrui» potesse trasferire l'atto di proprietà della cappella immediatamente, a prescindere dal fatto che, sfortunatamente, non fosse stata terminata, mettendo in chiaro che il terreno era stato donato dal Presidente della Camera Municipale, il colonnello Manoel Maximiano Junqueira, ma che tutte le suppellettili religiose avrebbero dovuto essere restituite a lui, quando ne fosse stata

\footnotetext{
${ }^{30}$ ADDLS, Libro dei registri, scritti e altri ordini emanati dalla Santa Sede apostolica e dalla nunziatura, 18881929 , p. 60.

${ }^{31}$ Il riferimento è all'associazione Associação dos Catequistas Voluntários, successivamente denominata Sociedade Legião Brasileira de Cultura e Civismo, fondata da padre Euclides [N.d.T.].

$3^{2}$ ADDLS, Libro dei registri, degli scritti e degli altri ordini emanati dalla Santa Sede apostolica e dalla nunziatura, 18881929, p. 60.
} 
fatta richiesta, per comporre la sua cappella privata, che sarebbe stata realizzata nella sua fazenda.

L'elenco dei beni si componeva delle immagini di San Manuele e di Santa Amelia, provenienti dal Portogallo e oramai strumenti utilizzati per la celebrazione eucaristica33. Si sottolineava che c'era l'intenzione di passare la mano in merito al controllo diretto della cappella, ma che, se le "faccende religiose" non fossero andate per il verso auspicato, si sarebbe potuta richiedere la restituzione dei beni non trasferiti al potere ecclesiastico.

San Manuele e Santa Amelia erano icone di proprietà dello stesso Manoel Maximiano Junqueira e di Amélia Junqueira e si trovavano presso l'altare per un ragione simbolica. L'utilizzazione degli onomastici era una modalità usuale delle famiglie influenti per creare una commistione tra il loro potere e l'alone di santità che si generava nel chiamare le loro fazende con nomi di santi che corrispondevano ai loro, indipendentemente dal fatto che esistessero o no; anche perché la Chiesa opponeva una scarsa resistenza, collaborando alla mistificazione portata avanti dagli onorati benefattori dei loro confratelli dal momento che anche questo faceva parte della tradizione lusitana.

Non è stata infatti trovata la documentazione che attesti il trasferimento della proprietà della cappella, dal momento che era e restava la proprietà di un privato. Così come la costruzione della cappella - nel 1905, quando si costruivano anche la chiesa degli Agostiniani e la chiesa madre, e futura cattedrale - si configurava come parte del progetto di consolidamento dell'influenza della Chiesa sul potere locale, allo stesso modo la proprietà privata della cappella evidenziava una disputa economica che si perpetuò per decenni. Si diceva che San Sebastiano non fosse uguale a Sant'Antonio. Quest'ultimo fu prolifico nell'attirare fedeli e donazioni facendo divenire la Chiesa di Sant'Antonio più lucrativa della stessa cattedrale.

La concorrenza tra Sant'Antonio e San Sebastiano a Ribeirão Preto - ma non solamente lì - andava aldilà della rappresentazione lusitana e ultramontana. La sua divisione coinvolgeva il cattolicesimo devozionale, dove il clero legato a San Sebastaino perdeva nella concorrenza con coloro che erano legati a Sant'Antonio. Il Santo dei poveri era popolare tra persone molto differenti tra loro che ne erano devote: cuoco e miracoloso nella produzione dei pani attraeva e seppe attrarre moltitudini in cerca di una pagnotta per garantirsi di che sfamarsi durante tutto l'anno. La Chiesa rafforzò ancora più questa devozione distribuendo settimanalmente pani nelle mani dei poveri

33 ADDLS, Busta Ribeirão Preto (II). Lettera di Alfredo V. P. de Souza a Antônio P. Reimão, del 4 ottobre 1905 . 
come sopravvivenza di una tradizione caritatevole medioevale. La sovrapposizione con la diceria non cristiana di propiziatore di matrimoni gli procurò un gran numero di devote e il suo ruolo di taumaturgo completava la triade di doti fondamentali a conquistare la devozione presso persone differenti fra loro e che facevano sì che attraesse un numero sempre maggiore di devoti e donazioni.

Per altri versi il controllo parziale del clero sulla cappella dimostrava la vittoria dell'etnia italiana e dell'ultramontanismo, benché vacillante, a detrimento dei Portoghesi. Gli immigranti italiani giungevano a migliaia e conquistavano il controllo del bairro di Barracão e la preminenza all'interno della cappella favorendo il consolidamento dell'ultramontanismo ed esigevano preti che sostenessero questo orientamento. La risposta a questa esigenza furono gli scalabriniani.

\section{Gli scalabriniani nella diocesi di San Sebastiano di Ribeirão Preto}

Benché avessero iniziato la diffusione della pastorale dell'immigrante a partire dalla capitale, San Paolo, dove stabilirono la sede principale della Congregazione finirono per trasferirla nella città di Ribeirão Preto, nel quadro della riorganizzazione interna o nel tentativo di ridurre la riprovazione generale che si era generata con lo scandalo conosciuto come caso Idalina e che rischiava di dimostrare pubblicamente che l'ultramontanismo e la romanizzazione non avevano ottenuto i loro obiettivi nella Chiesa.

Idalina era ospitata all'interno dell'orfanato Cristoforo Colombo, gestito dalla Congregazione dei Missionari di San Carlo a San Paolo. Secondo le accuse degli anarchici questa minorenne sarebbe stata stuprata da un prete e assassinata dal superiore della Congregazione. Secondo la versione della Chiesa, la bambina fu consegnata a una signora che si sarebbe identificata come la madre. Tuttavia i genitori della minorenne erano spariti. Il dibattito sul consolidamento del processo migratorio e della pastorale dell'immigrante è stato sviluppato in Anarquismo, Estado e pastoral do imigrante ${ }^{34}$.

Dal momento che il caso si sviluppò nella seconda metà del primo decennio del Novecento, ma cadde in prescrizione l'imputazione del crimine, i missionari si erano dedicati a svincolare l'immagine della Congregazione a quella l'orfanatrofio, che era un elemento fondamentale della strategia della pastorale dell'immigrante. Così nel 1911 la

34 De SOUZA, Wlaumir Donisete, Anarquismo, Estado e pastoral do imigrante. Das disputas ideológicas pelo imigrante aos limites da ordem o Caso Idalina, São Paulo, Editora Uniesp, 2000. 
sede della Congregazione venne trasferita presso la cappella di Sant'Antonio "dei poveri” di Ribeirão Preto, ritornando all'antica sede della capitale dello Stato solo nel 1919.

Il trasferimento della sede della Congregazione a Ribeirão Preto era legata anche allo sviluppo dell'industria caffearia ad alta concentrazione di immigrati nell'Oeste Paulista. Con l'avanzamento della frontiera agricola dal Velho Oeste al Novo Oeste fu necessario decentralizzare l'azione missionaria per raggiungere più rapidamente gli immigrati.

In precedenza la prossimità di San Paolo con le aree contraddistinte da una maggior concentrazione di Italiani, rendeva possibile un lavoro efficace per gli Scalabriniani che potevano evangelizzare Campinas, Rio Claro, Limeira e altre città del Velho Oeste. Con il processo di spostamento della produzione del caffè e della manodopera addetta alla sua coltivazione, anche i missionari di San Carlo si spostarono.

La distanza dalla capitale dello Stato rispetto a Ribeirão Preto, la capitale dell'Oeste, imponeva l'apertura di una residenza, ma non il trasferimento della sede. Secondo i documenti della Congregazione, redatti da Don Scalabrini, era prevista la realizzazione di residenze all'interno del territorio e prossime ai centri ad alta concentrazione di immigrati in modo da garantire l'accesso alla religione e il mantenimento della dottrina cattolica ultramontana e romanizzata.

Sommata alle distanze, la concentrazione del caffè e degli immigrati nella regione risultò un elemento attrattivo per l'installazione della diocesi di San Sebastiano di Riberão Preto, nel 1907, dal momento che Don Alberto José Gonçalves, navigato politico del Partito Repubblicano del Paraná ne fu il primo vescovo, il che rappresentava una contraddizione dei principi ultramontani, che condannavano la partecipazione politica del clero; con Leone XIII, del resto era possibile parlare di un neoultramontanismo 35 .

Nei documenti contenuti nell'Archivio della sede arcidiocesana di San Paolo, nel 1905 Ribeirão Preto era definita come un buon luogo per la futura sede episcopale per via delle buone condizioni delle comunicazioni e dei trasporti ${ }^{36}$ grazie alle ferrovie che lo legavano la città, non solo con altri municipi, ma anche con le fazende, facilitando la possibilità per la Chiesa - così come per gli anarchici - di raggiungere gli immigrati.

La fondazione della diocesi era uno degli elementi rivelatori dell'ascesa al potere economico e dell'influenza della regione di Ribeirão Preto, dedita alla caffeicoltura, che

\footnotetext{
35 Ibidem.

${ }^{36}$ ADDLS, Busta Ribeirão Preto (II). Lettera di Francesco Curti per il vicario generale del 24 giugno 1905.
} 
aveva il soprannome di capitale dell'Oeste, Eldorado, nella misura in cui aveva costruito tutto un immaginario sulla prosperità del luogo e dei suoi abitanti. Molti di questi avevano trasferito una parte delle loro fortune dalle regioni in cui la caffeicoltura era in fase decadente, come quelle di Vale do Paraiba o di Velho Oeste, oltre ai caffeicoltori del Minas Gerais e agli agricoltori di altri Stati, alcuni persino dal Nordeste del Brasile, dal momento che si erano estese le relazioni di interdipendenenza del potere locale con quello dello Stato37.

Nel ruolo di vescovo diocesano, Don Alberto José Gonçalves aveva bisogno di dotarsi di un clero che permettesse la concretizzazione del progetto della Chiesa volto a garantire la sua influenza ultramontana nella regione. Per questa ragione varie congregazioni furono invitate, e altre si offrirono, per installarsi nella nuova diocesi. Tra le congregazioni che accorsero in città annoveriamo gli Scalabriniani.

Tutti i religiosi che giungevano erano avidi di sostegni economici che potessero realizzare i loro progetti ed edifici. Il vescovo era intento alla conclusione della costruzione della cattedrale, l'antica chiesa madre di San Sebastiano, il patrono del Portogallo; gli Scalabriniani nella manutenzione della cappella destinata al culto di Sant'Antonio, nato in Portogallo e comunemente soprannominato "dei poveri” e alla realizzazione di una scuola e di un ospedale destinati agli immigrati e tutti desideravano una buona sede.

\section{Conclusioni}

All'oligarchia piaceva l'idea di essere impegnata in quest'opera assieme agli immigrati, dal momento che, in questo modo, poteva garantire l'assistenza religiosa: nello stesso modo in cui permetteva un'assistenza agli invalidi, forniva un motivo in più agli immigrati per dirigersi a Ribeirão Preto.

La fondazione della cappella di Sant'Antonio dei poveri legittimava il potere dell'oligarchia che si era legata al potere locale nella persona del Colonnello M. M. Junqueira, mentre la sua trasformazione in una cappella destinata alla pastorale degli immigrati portava con sé la modalità aggregativa degli Italiani in un unico luogo: la chiesa.

In questo modo la chiesa diveniva il maggior punto di incontro degli immigranti a cui non poteva essere impedito di riunirsi dal momento che lo facevano in nome della

37 ELIAS, Norbert, O processo civilizador. Uma história dos costumes, vol. 2, Rio de Janeiro, Zahar, 1993, passim [ed. it., Il processo di civilizzazione, Bologna, Il Mulino, 1996]. 
fede, senza nessuna implicazione politica dichiarata38. Mentre l'immigrante proveniente dall'Italia non si vedeva come Italiano, la religione lo univa agli altri, rendendo possibile una costruzione dell'etnicità a partire dalla retorica di formazione di un patrimonio linguistico condiviso che rendesse possibile la ccoesione del gruppo anche aldilà degli steccati della fazenda39.

La Chiesa organizzava e conseguentemente strumentalizzava l'immigrante italiano per consolidare il suo potere assieme al blocco egemonico: questo la metteva sempre più nelle mire degli anarchici che si disputavano con quest'ultima il sostegno dei migranti, che si traduceva in influenza politica per determinare il futuro del paese.

Per eludere il controllo istituzionale promosso dallo Stato in quanto prolungamento della proprietà privata capitalista, talvolta i sindacati si trasformavano in circoli sociali o associazioni di beneficenza, che apparivano pubblicamente come costituiti per una finalità, ma che in pratica rendevano possibile l'incontro fra interessi comuni che andassero oltre la carità o il divertimento, permettendo il dibattito politico sugli interessi in gioco.

Si deve prendere in considerazione il fatto che i missionari di San Carlo riuscirono a creare il loro bollettino, benché all'ombra della diocesi - perché si trattava di una Pia Unione Diocesana e questo era il loro organo ufficiale - subito dopo l’inizio della loro azione nella diocesi di Ribeirão Preto, mentre invece il governo dicoesano riuscirà a creare il proprio solamente molti anni più tardi. Questo fatto evidenzia come gli immigrati legati alla devozione antoniana fossero molto più preoccupati della loro devozione che della sorte del processo di romanizzazione e del suo baluardo, la diocesi, che si muoveva su impulso dell'oligarchia locale, che da essa traeva profitto e beneficio politico.

La pubblicazione del bollettino, più che una rappresentazione dell'influenza del cattolicesimo dell'immigrante italiano era l'imposizione del potere economico che da esso si originava e di cui l'immigrante, isolatamente, non godeva, ma che nella cattolicità era sfruttabile: era la vittoria - a livello economico - dello straniero sull'autoctono.

Come la ricchezza derivata dal caffè fu in grado di attrarre la pastorale dell'immigrante, allo stesso modo, la mancanza di una cultura comune li allontanò da Ribeirão Preto. L'allontanamento degli Scalabriniani fu direttamente legato al fatto di aver previsto una grande crisi del caffè allorquando, all'epoca, si ragionava

${ }_{38}$ RIBEIRO, Maria Therezinha Janine, Desejado e temido. Preconceito contra o imigrante italiano na Primeira República, Tesi di Master in Storia, Facoltà di Filosofia, Lettere e Scienze umane dell'Universidade de São Paulo, São Paulo, 1985, pp. 97-99.

39 Ibidem. 
sull'eliminazione del prodotto in eccedenza. A ciò si aggiunse il fatto che il caso Idalina era giunto a conclusione, dal momento che - a partire dal 1919 - era caduto in prescrizione, rendendo così impossibile procedere nei confronti degli accusati. 


\section{* L'autore}

Wlaumir Donisete De Souza è Professore presso il Centro Universitario Barão de Mauá di Ribeirão Preto. Dopo essersi laureato in Filosofia alla PUC di Belo Horizonte e avere conseguito la specializzazione in Storia presso la UNESP, si è addottorato in Sociologia presso lo stesso ateneo.

URL: < http://www.studistorici.com/progett/autori/\#DeSouza_Wlaumir >

\section{Per citare questo articolo:}

De SOUZA, Wlaumir Donisete, «Immigrazione e contesa politico-religiosa nella Prima Repubblica: la Cappella di Sant'Antonio dei poveri a Ribeirão Preto», Diacronie. Studi di Storia Contemporanea : "Se creare è definire", 29/03/2016,

URL:< http://www.studistorici.com/2016/03/29/desouza_numero_25/ >

\section{Diacronie Studi di Storia Contemporanea $\widehat{\beta}$ www.diacronie.it}

Risorsa digitale indipendente a carattere storiografico. Uscita trimestrale. redazione.diacronie@hotmail.it

Comitato di redazione: Jacopo Bassi - Luca Bufarale - Elisa Grandi - Antonio César Moreno Cantano - Deborah Paci - Fausto Pietrancosta - Alessandro Salvador - Matteo Tomasoni - Luca Zuccolo

Diritti: gli articoli di Diacronie. Studi di Storia Contemporanea sono pubblicati sotto licenza Creative Commons 3.0. Possono essere riprodotti e modificati a patto di indicare eventuali modifiche dei contenuti, di riconoscere la paternità dell'opera e di condividerla allo stesso modo. La citazione di estratti è comunque sempre autorizzata, nei limiti previsti dalla legge. 